\title{
Editorial zum Themenschwerpunkt Knorpelchirurgie
}

Sehr geehrte Leserin, sehr geehrter Leser, liebe Kolleginnen und Kollegen!

Knorpelschäden des Gelenkknorpels stellen unabhängig von ihrer Ätiologie nach wie vor ein Problem dar. Sie heilen nicht, wie das von anderen Geweben mit mehr oder weniger stark ausgeprägter regenerativer Kapazität her bekannt ist.

Hunter stellte bereits 1743 fest: „From Hippocrates down to the present age, we shall find, that an ulcerated cartilage is universally allowed to be a very troublesome disease; ...; and that, when destroyed, it is never recovered" [4].

In der deutschen Literatur findet man im „Handbuch der Gewebelehre des Menschen “ von Kölliker 1852 die Bestätigung: „Wiedererzeugungsfähigkeit besitzen Knorpel nicht, und ebenso wenig heilen Knorpelwunden durch Knorpelsubstanz" [5].

Diese Erkenntnis wurde in den letzten beiden Jahrhunderten mit neuen Untersuchungstechniken immer wieder experimentell bestätigt. Gelenkknorpel ist ein ganz besonders belastbares Gewebe, das uns im Normalfall durch seine biomechanischen Eigenschaften (es hat einen geringeren Reibungskoeffizienten als Eis auf Eis) die Mobilität bis ins hohe Alter erlaubt. Ist die mechanische Belastbarkeit des Gelenks durch eine Knorpelverletzung gestört, kommt es zum verstärkten Verschleiß der verbleibenden Areale und damit zur Arthrose.

Die Operative Orthopädie und Traumatologie hat es sich deshalb in diesem Themenschwerpunkt zur Aufgabe gemacht, die publizierten Techniken zur Behandlung von Knorpeldefekten, 2001 von Mats Brittberg begonnen [1], fortzuführen.

Stand damals die zellbasierte Therapie deutlich im Vordergrund des Interesses, so wurde sie kurze Zeit später von unterschiedlichsten Variationen der osteochondralen Transplantationen flankiert:

Schoettle \& Imhoff beschrieben 2002 die OATS am Talus über eine Chevron-förmige Innenknöchelosteotomie [8]; Horas \& Schnettler sowie Meenen \& Rischke folgten kurz darauf mit der Darstellung der osteochondralen Zylindertransplantation am Kniegelenk mit einem Diamantschleifsystem [3, 6]; Steinwachs \& Kreuz publizierten 2004 einen erweiterten Zugang zur Behandlung osteochondraler Defekte am Talus [9]; auch computerassistierte Operationen zur Therapie der Osteochondrosis dissecans am Talus lassen sich nachlesen [7], und eine Sonderform ist sicher die osteochondrale Transplantation am Femurkondylus mit frischem Allograft [2].

Im vorliegenden Heft sind neben der osteochondralen Transplantation von „Megafragmenten“ wieder zwei Publikationen über die zellbasierte Therapie zu lesen. Entsprechend der zunehmenden Entwicklung und Anwendung des ,tissue engineering“ in der Orthopädie runden diese Beiträge das Feld der Knorpeltherapie ab. Wir sind gespannt, was uns die Zukunft bringen wird.

Als Herausgeber dieser Beiträge zur Knorpeltherapie ist mir wieder aufgefallen, wie schwer es ist, langfristig aussagekräftige Ergebnisse zu erhalten. Es ist den Autoren dieses Themenschwerpunkts zu danken, dass sie dies durchweg versucht haben. Die Entwicklungen auf dem Gebiet sind so schnell, dass sich die Methoden bereits geändert haben, wenn die Studiengröße eine kritische Menge erreichen könnte. So muss man leider immer wieder zu der Empfehlung kommen, die Therapie von Knorpeldefekten nur in spezialisierten Zentren durchzuführen, die sich mit der Thematik ernsthaft und nachhaltig beschäftigen (wozu nach meinem Verständnis auch prospektiv angelegte Beobachtungen der Patienten zählen).

Ich wünsche den Leserinnen und Lesern viel Freude bei der Lektüre. Nur wer sich informiert, kann die richtigen Entscheidungen treffen.

\section{Prof. Dr. Maximilian Rudert,}

\section{Literatur}

Herausgeber

1. Brittberg M. Die Transplantation autogener Knorpelzellen in Gelenkflächendefekte des Kniegelenks. Oper Orthop Traumatol 2001;9:185-94.

2. Fischer $M$, Koller $U$, Krismer M. Die Verwendung von frischen Allografts bei der Osteochondrosis dissecans am lateralen Femurkondylus. Oper Orthop Traumatol 2006;18:245-58.

3. Horas U, Schnettler R. Knorpeldefekte am Kniegelenk und autogene osteochondrale Zylindertransplantation. Oper Orthop Traumatol 2002;10: $220-34$.

4. Hunter W. On the structure and diseases of articulating cartilages. Philos Trans Roy Soc 1743;42:514-21.

5. Kölliker A.: Handbuch der Gewebelehre des Menschen. Leipzig: Engelmann, 1867:64-9.

6. Meenen NM, Rischke B. Autogene osteochondrale Transplantation (AOT) bei Knorpeldefekten am Femurkondylus. Oper Orthop Traumatol 2003;15:38-56.

7. Rosenberger RE, Fink C, Bale RJ, et al. Computer-assisted minimally invasive treatment of osteochondrosis dissecans of the talus. Oper Orthop Traumatol 2006;18:300-16.

8. Schoettle PB, Imhoff AB: Die osteochondrale Autograft-Transplantation (OATS) am Talus. Oper Orthop Traumatol 2002;14:123-40.

9. Steinwachs M, Kreuz PC. Die Behandlung von dorsalen osteochondralen Defekten des Talus über einen erweiterten Zugang. Oper Orthop Traumatol 2004;16:300-19.

Oper Orthop Traumatol 2008;20:187

DOI 10.1007/s00064-008-1300-4 